$\begin{array}{cl}\begin{array}{c}\text { Revue } \\ \text { de /histoire }\end{array} & \text { Revue de l'histoire des religions } \\ \text { des religions } & \begin{array}{l}\mathbf{3} \text { |2008 } \\ \text { Varia }\end{array}\end{array}$

\title{
La mort d'Aqhat : chasse et rites de passage à Ugarit
}

Aqhat's death: Hunting and rites of passage at Ugarit

Jean-Marie Husser

\section{OpenEdition}

Journals

Édition électronique

URL : http://journals.openedition.org/rhr/6833

DOI : $10.4000 /$ rhr.6833

ISSN : 2105-2573

Éditeur

Armand Colin

Édition imprimée

Date de publication : 1 septembre 2008

Pagination : 323-345

ISBN : 978-2200-92445-4

ISSN : 0035-1423

Référence électronique

Jean-Marie Husser, "La mort d'Aqhat : chasse et rites de passage à Ugarit », Revue de l'histoire des religions [En ligne], 3 | 2008, mis en ligne le 01 septembre 2011, consulté le 30 avril 2019. URL : http:// journals.openedition.org/rhr/6833; DOI : 10.4000/rhr.6833 


\section{La mort d'Aqhat : chasse et rites de passage à Ugarit}

L'existence de rites de passage dans les sociétés antiques du ProcheOrient demeure très difficile à cerner, car de telles pratiques ne sont pas attestées directement dans notre documentation. Par une approche comparative et la mise en évidence d'un code symbolique associant chasse et érotisme dans la légende ougaritique d'Aqhat, on pense avoir réuni un faisceau d'indices suffisant pour affirmer l'existence d'un rite de passage à Ougarit. L'hypothèse que nous développons ici est que la mort du héros et le différend qui l'oppose à la déesse prennent leur pleine signification dans le contexte d'un rite de passage des jeunes gens à l'âge adulte. La mort d'Aqhat apparaît comme un contre-exemple hérö̈que et le récit paradigmatique de l'échec de ce passage.

\section{Aqhat's death : Hunting and rites of passage at Ugarit}

The existence of rites of passage in the societies of the Ancient Near East remains something very difficult to evaluate, because such practices are not mentioned directly in our documentation. From a comparative approach, and the discovery of a code of symbols that associates the hunt with eroticism in the Ugaritic legend of Aqhat, a sufficient set of evidence has been gathered to confirm the existence of such a rite of passage in Ugarit. In this article, we develop the hypothesis that the death of Aqhat and the quarrel in which he is opposed to the goddess find their full significance in the context of a rite of passage from youth to manhood. The death of Aqhat seems to be a heroic counter-example and the paradigmatic tale for the failure to achieve this passage successfully. 
De manière générale, la chasse est une activité qui manifeste, à travers les siècles et les cultures, une remarquable capacité à légitimer une appartenance sociale et à signifier la domination exercée sur un territoire par la force qu' on y déploie. Sollicitant des vertus considérées comme spécifiquement masculines de force, de courage, d'adresse dans la maniement des armes, elle apparaît aussi comme un exercice à haute valeur symbolique, et cela de multiples façons. La posture du chasseur à l'affût de sa proie, ainsi que le maniement des armes de jet fournissent un répertoire quasi universel d'expressions métaphoriques pour dire la quête sexuelle et le jeu érotique. Mais en même temps, la chasse engage l'homme dans le domaine inquiétant de la nature sauvage et du monde liminal, peuplé d'animaux dangereux et d'êtres hybrides qui nourrissent l'imaginaire mythique. De ce fait, l'aventure cynégétique est aussi le lieu d'une instabilité fondamentale qui se caractérise notamment par la réversibilité des situations, pour reprendre l'expression d'Alain Schnapp ${ }^{1}$ : le chasseur peut être à son tour poursuivi par l'animal et de prédateur, devenir soudainement la proie.

Cette possible inversion des rôles apparait précisément comme l'élément structurant du mythème du chasseur tué, si fréquent dans les mythologies antiques du bassin méditerranéen ${ }^{2}$. Dans son étude sur les « aspects orientaux » du mythe grec d'Adonis, Sergio Ribichini a justement souligné la prégnance de ce thème dans les restes épars d'une mythologie syro-palestinienne de la chasse, dont les textes d'Ougarit nous ont conservé les fragments les plus explicites ${ }^{3}$.

1. Alain Schnapp, « Chasse. Ses héros et ses mythes en Grèce ancienne », Dictionnaire des mythologies, dir. Yves Bonnefoy, Paris, Flammarion, 1999, p. 300-303.

2. Voir la synthèse classique sur le sujet de Joseph E. Fontenrose, Orion, the Myth of the Hunter and the Huntress, Berkeley-Los Angeles-London, University of California Press, 1981, et antérieurement, s'agissant du ProcheOrient ancien, les remarques de Paolo Xella, Problemi del mito nel Vicino Oriente antico, AION 36 (Suppl. 7), Napoli, 1976, p. 61-91.

3. Sergio Ribichini, Adonis. Aspetti «orientali» di un mito greco, Roma, CNR, 1981, p. 108-123. Enumérant l'épisode de la mort d'Aqhat (p. 111-113), le texte «Baal et les Voraces » (p. 113 s.), ainsi que la brève notice de Philon de Byblos sur la mort d'Hypsistos-Eliun tué par des bêtes sauvages (p. 114 s.), il conclut à la possibilité «d'individuare sempre più chiaramente in area siropalestinese una particolare mitologia della caccia, che contempla, probabilmente con sue funzioni specifiche, il tema del cacciatore fallito...» (p. 115). 
Dès lors que l'on reconnaît l'existence d'une telle mythologie dans cette partie du Proche-Orient ancien, la question se pose de savoir à quels codes symboliques et à quelles pratiques sociales renvoient ces récits. La mythologie ougaritique - souvent sollicitée, car la seule jusqu'ici conservée - est-elle en mesure, en dépit de son caractère très fragmentaire, de nous fournir quelques indices d'éventuelles pratiques sociales susceptibles de donner du sens à ce motif mythologique du chasseur tué ?

Cet article voudrait poursuivre en ce sens l'investigation menée par S. Ribichini en tentant d'accéder à la dimension anthropologique sous-jacente à cette « mitologia della caccia ». Précisons cependant que la documentation ougaritique n'a pas livré jusqu'ici de véritable « mythe de chasse », tel qu' on en connaît chez les peuples de chasseurs. Ces récits traditionnels fondent les règles de conduite que le chasseur doit observer avant, pendant et après son expédition de chasse, tant envers le gibier qu'envers les êtres surnaturels dont dépendent les animaux qu'il poursuit et les territoires qu'il parcourt. Dans les textes dont nous disposons, nous ne rencontrons que quelques épisodes épars liés au monde de la chasse et intégrés à la trame narrative de récits dont la finalité et l'horizon idéologique sont tout autres. Qu'il s'agisse de fragments de mythes archaïques réutilisés dans des compositions nouvelles, c'est vraisemblable, mais difficilement vérifiable et de peu d'importance pour notre propos $^{4}$. Ce que nous considérons, c'est la signification de la chasse dans la société du Levant à la fin du Bronze et les comportements qu'elle induit dans ce contexte ${ }^{5}$.

4. Sur la question de l'acculturation de mythes archaïques de chasse dans la société hittite, voir les remarques de Paolo Xella, « Remarques comparatives sur le "Roman de Kešši” », RHA 36, 1978, p. 215-224.

5. J'exprime ma gratitude à Nicolas Wyatt et Wilfred G.E. Watson qui ont bien voulu relire ces pages et me faire part de leurs remarques, ainsi qu'aux collègues de l'UMR 7044, notamment Thierry Petit et Daniela Lefèvre. 


\section{UN RITE DE PASSAGE COMME CONTEXTE AU RÉCIT DE LA MORT D'AQHAT ?}

Dans les textes ougaritiques, le motif du chasseur tué apparaît une première fois dans le mythe dit de «Baal et les Voraces » (KTU 1.12), dont la tablette est malheureusement très abîmée et la lecture pleine d'incertitudes ${ }^{6}$. On y lit comment Baal va à la chasse « aux confins de la steppe » (i 35 : pi'atu malbari) où il rencontre les « Voraces » ('âkilüma//`āqiqūma), des êtres hybrides et démoniaques nés dans cette contrée sauvage. À la suite de circonstances impossibles à déchiffrer, Baal posté à l'affût se trouve être la victime de ces Voraces et succombe sous leurs coups : «Oui, Baal est tombé, comme un taureau, Haddu est sur les genoux, tel un taurillon au milieu de la mêlée / du marécage ${ }^{7}$. Le chasseur est à son tour devenu le gibier de ceux qu'il traquait et l'inversion des situations se trouve encore soulignée par la comparaison de la mort de Baal avec celle d'un taureau. L'état matériel du texte et l'absence de contexte rendent cependant l'interprétation de ce mythe difficile et sa valeur documentaire pour une mythologie de la chasse reste limitée.

C'est bien dans la Légende d'Aqhat que l'on trouve l'expression la plus développée et la plus explicite du mythème du chasseur tué 8 . Dans la seconde partie du texte conservé de ce poème épique, le jeune Aqhat meurt à la chasse, tué par un faucon. L'oiseau de proie fut envoyé par la déesse Anat en punition de l'affront que lui a fait le

6. Par commodité, les textes ougaritiques seront cités d'après l'édition manuelle de Manfried Dietrich, Oswald Loretz, Joaquín Sanmartín, The Cuneiform Alphabetic Texts from Ugarit, Ras Ibn Hani and Other Places, Münster, Ugarit-Verlag, $1995\left(K T U^{2}\right)$. Deux traductions de référence sont proposées ici : André Caquot, Maurice Sznycer, Andrée Herdner, Textes ougaritiques. Tome I : Mythes et légendes, LAPO 7, Paris, Le Cerf, 1974, p. 315-351 ; Nicolas Wyatt, Religious Texts from Ugarit, Sheffield, Academic Press, 1998, p. 162-168. Voir la dernière étude très complète de ce texte dans Manfred Dietrich, Oswald Loretz, Studien zu den ugaritischen Texten. I, Mythos und Ritual in KTU 1.12, 1.24, 1.96, 1.100 und 1.114, AOAT 269/1, Münster, Ugarit-Verlag, 2000, p. 1-141.

7. KTU 1.12 : ii 53-55: kn. npl. $b^{c} l / k m$ tr wtkms. hd $/ \mathrm{km}$. ibr. btk. mšmš. Pour la traduction de ce dernier terme, voir Dietrich-Loretz, op. cit. (note 6), p. 65.

8. Texte dans $K T U 1.17$ : vi - 1.18 : iv ; traduction dans Caquot-SznycerHerdner, op. cit. (note 6), p. 430-440, et Wyatt, op. cit. (note 6), p. 270-287. 
jeune homme en refusant de lui donner son arc. Ce texte présente donc l'intérêt de mettre en scène un jeune héros de rang royal et une déesse dont les liens avec la guerre et la chasse d'une part, avec la sexualité d'autre part, sont bien attestés. Le contenu narratif et le genre épique de ce récit réfèrent clairement aux valeurs et aux codes sociaux d'une aristocratie palatiale de la fin de l'Age du Bronze dont ils contribuent à structurer l'idéologie. Dans ce contexte social précis, l'épisode de la mort d'Aqhat apparait comme la mise en récit d'un contre-exemple héroïque, d'un échec dans la relation qui devait s'établir entre le jeune homme et la déesse au moment où il accède à la vie d'adulte.

L'hypothèse que nous développons ici est que la mort d'Aqhat et le différend qui l'oppose à la déesse prennent leur pleine signification dans le contexte d'un rite de passage à l'âge adulte où la pratique de la chasse sert à la fois d'épreuve et de code symbolique à ce passage. Il faut souligner d'emblée que la documentation ougaritique ne livre aucune information explicite sur l'existence de rites de passage tels que l'entend l'anthropologie sociale. Devant cet apparent silence des textes, l'enquête ne peut s'appuyer que sur un faisceau d'indices convergents. Il ne s'agit certes pas de prétendre que la Légende d'Aqhat concerne directement un tel rite, ni d'en faire un récit initiatique, même si l'éducation du jeune homme semble être un thème-clé de la première partie du poème, comme en témoigne la quadruple répétition du «Catalogue des devoirs du fils idéal » $(K T U 1.17)^{9}$. On tentera simplement de montrer que l'épisode de la mort d'Aqhat suggère l'existence d'un tel schéma rituel par la référence qui y est faite à un code symbolique propre à structurer l'intégration du jeune homme dans la classe des hommes adultes, en procédant par une inversion logique des signes du masculin et du féminin. Cela n'implique pas nécessairement l'existence d'un rituel complexe et actuellement impossible à décrire ; mais cela suppose au moins que l'activité cynégétique, pour la classe dominante à Ugarit, offrait un cadre institutionnel et remplissait une fonction symbolique analogues à ce qui est observé dans d'autres sociétés antiques du pourtour méditerranéen. Une approche comparative s'impose donc

9. Voir M. J. Boda, «Ideal Sonship in Ugarit », UF 25, 1993, p. 9-24. 
ici, compte tenu de la nature fragmentaire de la documentation, mais aussi de la dimension anthropologique de cette question.

Les analogies entre le sort tragique d'Aqhat et celui des héros grecs Actéon, Orion, Hippolyte ou Atalante ont été maintes fois rappelées ${ }^{10}$. Le rôle de la sexualité juvénile a en particulier été souligné dans tous ces mythes où interviennent tour à tour les déesse Artémis et Aphrodite qui entretiennent avec ces héros des rapports tissés d'ambiguïté. Les territoires de chasse apparaissent comme un espace aux marges de la cité où les règles sociales définissant les rapports entre les sexes sont susceptibles de transgression. Mais l'éveil du désir sexuel introduit le trouble dans les règles qui régissent les comportements du jeune chasseur dans cet espace sauvage, car l'érotisme est réputé mettre en danger la complicité qui le lie au monde animal.

La fonction initiatique de la chasse dans l'accession des jeunes gens à l'âge adulte dans la Grèce archaïque et classique a fait l'objet de travaux bien connus d'histoire et d'anthropologie des religions depuis l'impulsion décisive donnée par Henri Jeanmaire et Angelo Brelich ${ }^{11}$. Quelques publications récentes ont poursuivi la recherche dans les mondes égéens et hittites du Bonze Récent et montré un schéma comparable ${ }^{12}$. Dès que l'on aborde le Proche-

10. Notamment par J. E. Fontenrose, op. cit. (note 2), p. 217-220.

11. En particulier Walter Burkert, Structure and History in Greek Mythology and Ritual, Berkeley, 1979, p. 118-122 ; Pierre Vidal-Naquet, « Le chasseur noir et l'origine de l'éphébie athénienne », Le Chasseur noir, Paris, (Maspéro, 1981) La Découverte, 1991, p. 151-175 ; «Le cru, l'enfant et le cuit », ibid. p. 177-207; Alain Schnapp, Le Chasseur et la cité. Chasse et érotique dans la Grèce ancienne, Paris, Albin Michel, 1997 ; Robin MitchellBoyask, «Euripides' Hippolytus and the Trial of Manhood», Rites of Passage in Ancient Greece : Literature, Religion, Society, Mark W. Padilla (ed.), Lewisburg (Penn.)-London, Bucknell-Associated University Press, 1999, p. 43-66.

12. Christine E. Morris, «In Pursuit of the White Tusked Boar : Aspects of Hunting in Mycenaean Society », Celebrations of Death and Divinity in the Bronze Age Argolid, R. Hägg - G. C. Nordquist (eds), ActaAth-4 ${ }^{\circ}$, 40, Stockholm, 1990, p. 149-156 ; Nanno Marinatos, « Striding Across Boundaries : Hermes and Aphrodite as Gods of Initiation », Initiation in Ancient Greek Rituals and Narratives, D. B. Dodd - C. A. Faraone (eds), Routledge, LondonNew York, 2003, p. 130-151 ; Massimo Cultraro, « Exercise of Dominance. Boar Hunting in Mycenaean Religion and Hittite Royal Rituals », Offizielle Religion, locale Kulte und individuelle Religiosität, M. Hutter - S. HutterBraunsar (Hrg), AOAT 318, Münster, Ugarit-Verlag, 2004, p. 117-135. 
Orient ancien en revanche, la question des rites de passage disparait de l'horizon de la recherche. Il est significatif à cet égard que les deux monographies de Karel van der Toorn consacrées à la religion domestique ou familiale, ne comportent pas une ligne sur ce sujet en dehors des rites de mariage comme mode d'incorporation de la jeune femme dans le groupe de son mari ${ }^{13}$. Même constat dans l'enquête de David P. Wright sur les descriptions de rituels dans la Légende d'Aqhat ${ }^{14}$. L'auteur tente une explication de cette ritual failure qui survient lors du banquet où Anat demande son arc à Aqhat sans s'aventurer au-delà de la ritualité du repas lui-même. Pourtant, comme nous le verrons plus loin, les circonstances du récit suggèrent que ce banquet s'inscrit dans le cadre plus large de l'accession du jeune adolescent à l'âge adulte.

L'existence de rites de passage dans les sociétés du ProcheOrient ancien aux époques du Bronze et du Fer ne fait cependant guère de doute, même si l'investigation se heurte, ici encore plus que dans le domaine grec, à la difficulté offerte par une documentation désespérément fragmentaire. On a de bonnes raisons de penser, par exemple, que la circoncision pratiquée dans le judaïsme dès l'époque perse comme signe d'appartenance ethnique résulte de la transformation d'un antique rite pubertaire et prénuptial ${ }^{15}$. De même, l'épisode biblique de la fille de Jephté parcourant les montagnes avec ses compagnes pendant deux mois pour «pleurer sa virginité »

13. Karel van der Toorn, From Her Cradle to Her Grave, Sheffield, JSOT Press, 1994 ; Family Religion in Babylonia, Syria and Israel, Leiden, Brill, 1996. À propos de la puberté des jeunes filles, van der Toorn note simplement, p. 48 du premier ouvrage : "The first menstruation radically marked the transition. It is known that in many cultures this event gives rise to certain rites through which the young girl is incorporated into the women's world". On aurait souhaité en lire davantage.

14. David P. Wright, Ritual in Narrative. The Dynamic of Feasting, Mourning, and Retaliation Rites in the Ugaritic Tale of Aqhat, Winona Lake, Eisenbrauns, 2001, spéc. p. 100-122.

15. En tenant compte notamment de la péricope de Ex. 4,24-26. Voir en dernier lieu Howard Eilberg-Schwarz, The Savage in Judaism, Bloomington, 1990, pp. 141-148. La pratique d'une circoncision pré-maritale semble également connue à Ugarit, si l'on accepte l'interprétation de KTU 1.23 : 8-11 proposée par Nicolas Wyatt, « The Pruning of the Vine in KTU 1.23 », UF 24, 1992, p.426-430. 
(Juges 11,37-40), apparaît à l'évidence comme le mythe étiologique d'un ancien rite de puberté pour jeunes filles célébré annuellement ${ }^{16}$. Cette interprétation déjà ancienne d'un texte très laconique se trouve maintenant confortée par la comparaison avec les fresques minoennes de Thera-Akrotiri, dont Nanno Marinatos a récemment proposé une lecture tout à fait convaincante ${ }^{17}$. Cet ensemble pictural met visiblement en scène un rite de puberté où les jeunes filles sont envoyées dans la montagne, nues-pieds, pour y cueillir des crocus qu'elles offrent ensuite à la déesse. Après cette période de marge où elles doivent assumer leur premier sang, elles font l'objet d'une purification et sont réintégrées dans la citée.

La récente tentative de Christophe Lemardelé de retrouver, derrière le mythe biblique de Samson (Juges 13-16), une institution guerrière archaïque attire également notre attention ${ }^{18}$. Selon lui, « tous les indices relevés montrent la cohérence qui existe entre le héros Samson et une « institution » de nazir difficile à cerner, mais probablement liée à la guerre et aux jeunes guerriers, par notamment la chevelure sauvage. [...] La seule inconnue restant la nature précise du nazir : simple rite de passage des jeunes hommes ou «consécration » guerrière de quelques-uns ? ${ }^{19}$. Même si C. Lemardelé penche avec raison pour la seconde hypothèse s'agissant de la nature du nazir, la question d'éventuels rites de passage pour intégrer les jeunes hommes aux différentes catégories de guerriers dans l'Israël des débuts de la monarchie (nezirîm, bahurîm ou gibborîm) reste posée.

16. Et non comme la légende cultuelle du culte de Tammuz parmi les femmes israélites, comme le propose van der Toorn, op. cit. (note 13), p. 117.

17. Nanno Marinatos, Minoan Religion. Ritual, Image, and Symbol, University of South Carolina Press, 1993 ; chap. 10 : «Ritual contests, hunting, and rites of passage », p. 201-220.

18. Christophe Lemardelé, «Samson le nazir : un mythe du jeune guerrier », RHR 222, 2005, p. 259-286. L'argument a été développé dans une thèse de doctorat soutenue en 2007 à Paris (EPHE, Ve Section) : Etre «nazir» : du guerrier yahwiste au væu cultuel du judaïsme ancien.

19. Ibid. p. 284 s. 


\section{FonCtions SOCIALES de LA CHASSE À OUgARIT}

L'importance de la chasse dans la mise en scène de l'idéologie royale assyro-babylonienne est bien connue ${ }^{20}$. Il est évident également que la pratique cynégétique a, au moins dans l'esthétique et l'imaginaire, rempli un rôle semblable dans le monde Syro-palestinien des $\mathrm{II}^{\mathrm{e}}$ et $\mathrm{I}^{\mathrm{er}}$ millénaires, ainsi qu'en témoignent les nombreux sceaux et objets décorés de motifs de chasse retrouvés à Ugarit et ailleurs ${ }^{21}$. Comme dans les sociétés de la Mésopotamie, ce thème iconographique réfère à la royauté et contribue à sa symbolisation. De façon plus décorative, mais non moins significative, de nombreuses scènes de chasse ou figurations animales représentées sur divers supports illustrent la réversibilité du jeu cynégétique évoquée plus haut, l'un des thèmes majeurs de la mythologie de chasse. Le chasseur y devient proie, tandis que le gibier se transforme en bête féroce ; le lion, prédateur type, se trouve à son tour traqué par le chasseur auquel il sert de modèle, etc ${ }^{22}$.

Dans l'aire syro-palestinienne du Bronze Moyen au Fer II ( ca 2000-500 av. J.-C.) qui nous intéresse ici, la fonction et la signification de la chasse diffèrent selon qu'elle est pratiquée dans les régions urbanisées à production agricole ou dans les zones semi-arides

20. Voir Elena Cassin, «Le roi et le lion », Le Semblable et le différent, Paris, La Découverte, 1987, p. 167-213.

21. Notamment les panneaux d'ivoire du lit d'apparat retrouvés dans le palais d'Ugarit (voir Jacqueline Gachet-Bizollon, « Le panneau de lit en ivoire de la cour III du palais royal d'Ougarit », Syria 78, 2001, p. 19-82), ainsi que les deux coupes en or retrouvées sur l'acropole (RS 5.031 et 5.032 ; Claude Schaeffer, Ugaritica II, Paris, 1949, p. 1-48), auxquels font échos quelques spécimens de bols phéniciens du I ${ }^{\mathrm{er}}$ millénaire (Glenn Markoe, Phoenician Bronze and Silver Bowls from Cyprus and the Mediterranean, Classical Studies 26, Berkeley-Los Angeles, University of California Press, 1985, p. 67, 191, 278-279).

22. Voir, par exemple, la coupe en or ornée d'une chasse royale (RS 5.031; C. Schaeffer, Ugaritica II, Paris, 1949, p. 1-48) : le roi, debout sur un char tiré par deux chevaux, vise de son arc des animaux sauvages, une chèvre et trois taureaux. Comme l'a bien noté Marguerite Yon : «La composition circulaire fait que le vieux taureau, en tête des animaux, se retrouve derrière le char sur lequel il charge, tête baissée » (La Cité d'Ougarit, Paris, ERC, 1997, p. 175). 
où vivent des groupes tribaux d'éleveurs semi-nomades. Pour ces derniers, la chasse demeure une activité de protection des troupeaux et une source alimentaire non négligeable, l'animal d'élevage n'étant qu'exceptionnellement consommé. Pour les populations sédentaires et citadines des régions agricoles en revanche, elle a perdu ce rôle économique primaire au profit d'une fonction avant tout sociale. Si elle peut assumer encore occasionnellement la protection des animaux domestiques et des cultures, la chasse est devenue avant tout une activité sportive pratiquée par la classe dominante qui se met en scène à travers elle et l'intègre à des représentations symboliques.

Au témoignage des textes, l'apport de la chasse dans l'alimentation des Ougaritains semble insignifiant : la littérature épique (légendes de Keret et d'Aqhat) ne mentionne aucune consommation de gibier dans les repas aristocratiques ${ }^{23}$, où l'on mangeait essentiellement la viande des animaux domestiques. Cette observation est confirmée par les études d'archéozoologie pour l'ensemble de la région ${ }^{24}$. Il ne semble pas non plus que la chasse ait tenu un rôle quelconque dans l'économie du sacrifice: parmi les espèces animales sacrifiées, on ne relève aucun gibier, à l'exception possible de quelques oiseaux ${ }^{25}$. Dans les textes mythologiques, la table des festins des dieux est abondamment chargée de viandes, mais les animaux consommés sont les mêmes que ceux offerts dans le cadre du rite sacrificiel : bœufs, veaux gras, ovins. Sans surprise donc, la diète carnée des dieux s'avère être la même que celle des hommes de la classe dominante.

23. Cf. $K T U 1.15$ : iv 4 et $15 ; 1.17$ : ii 29 et v 17.

24. "Hunting was economically of no importance in the Bronze Age towns and cities of the Orient, in contrast to the preceding period of the Chalcolithic, in which some sites show that hunting could be a major source of animals products". A. T. Clason - H. Buitenhuis, "Change and Continuity in the Animal Food Resources in Bronze Age Towns of the Orient », Der orientalische Stadt: Kontinuität, Wandel, Bruch, G. Wilhelm (Hrg), CDOG 1, Saarbrücken, 1997, p. 199-218.

25. Voir le recensement détaillé des offrandes sacrificielles fait par Dennis Pardee, Les Textes rituels, RSO 12, 2 vol., Paris, ERC, 2000, p. 913-925. 
Deux exceptions cependant à cette observation générale : dans le récit mythologique de la mort de Baal, la déesse Anat accomplit un immense sacrifice funéraire après la sépulture du dieu (KTU 1.6 : i 19-29). Parmi les six espèces d'animaux sacrifiés, à part les bœufs ('alpüma) et les moutons (sa'nūma), quatre sont des espèces sauvages : des aurochs (rûmūma), des bouquetins (yaclūma), des daims (ayyalūma), des chevreuils (yaḥmarūma) ${ }^{26}$. L'énormité du sacrifice est à la mesure de la qualité du défunt, et à l'abondance des animaux sacrifiés - soixante-dix par espèce - s'ajoute la valeur intrinsèque des victimes. Il est probable que la présence de nombreux animaux sauvages ait pour but de relever le prestige de cet acte sacrificiel, mais elle est aussi la signature de celle qui offre le sacrifice. Anat, en effet, préside à l'activité cynégétique et apparaît nettement à Ugarit comme une figure de la potnia therôn ${ }^{27}$.

Seconde exception significative : Anat se retrouve, associée à Ashtart ${ }^{28}$, dans la tablette dite du «marzihu de El $(K T U 1.114)^{29}$. Ce texte para-mythologique décrit un festin offert par El aux autres dieux dans son palais et se termine en beuverie. Il n'est pas douteux que le récit présente la transposition mythologique d'une pratique

26. J'adopte la restitution du texte ligne 29 : [ttbb. šb ${ }^{c} m$. y] hmrm « [elle abat soixante-dix che]vreuils », proposée par H. Louis Ginsberg, «Interpreting Ugaritic Texts », JAOS 70, 1950, p. 156-160. Certains traducteurs préfèrent la lecture [ttbh . šb ${ }^{\mathrm{c}} \mathrm{m}$. ] $\mathrm{hmrm}$, «ânes », mais un sacrifice d'ânes paraît peu probable ici. L'âne n'est offert en sacrifice qu'en de rares occasions, lorsque l'intégrité de la cité est en jeu, semble-t-il ; de plus, les deux seules mentions d'un tel sacrifice (KTU $1.40: 26 ; 1.119: 16)$ utilisent le $\operatorname{mot}^{c} \hat{e} r u$, et non himāru.

27. Peggy L. Day, «Anat : Ugarit's "Mistress of Animals" », JNES 51, 1992, p. 181-190.

28. L'association de ces deux théonymes dans quelques textes (KTU $1.100: 20 ; 1.107: 39 ; 1.114: 9,22$ ) sous la forme composée Anat-Ashtart témoigne d'une phase initiale du processus qui conduira, au $\mathrm{I}^{\mathrm{er}}$ millénaire, d'une part à leur fusion dans la déesse syrienne Atargatis et, d'autre part, à l'assimilation d'Anat dans la figure de l'Astarté phénicienne.

29. Traduction : André Caquot, Textes ougaritiques II. Textes religieux et rituels, LAPO 14, Paris, Le Cerf, 1989, p. 71-78; Wyatt, op. cit. (note 6), p. 404-413. Pour un commentaire détaillé, voir Dennis Pardee, Les Textes para-mythologiques de la 24e campagne (1961), RSO IV, Paris, ERC, 1988, p.13-74 ; Dietrich - Loretz, op. cit. (note 6), p. 403-523. 


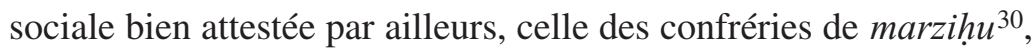
connues dans tout le Proche-Orient ancien pour leur grande consommation de vin. Plusieurs éléments évoquent ici sans doute possible un repas de chasse : le titre tout d'abord, qui parle clairement de venaison ${ }^{31}$; le comportement carnavalesque du dieu Yarikh, qui joue au «chien sous la table » (lignes 4-6), thème bien connu des repas aristocratiques; la présence enfin des déesses Anat et Ashtart (lignes 9-11), explicitement décrites dans leur rôle de chasseresses (lignes 22-23), et qui pourraient bien être les pourvoyeuses de ce banquet en viande fraîche. Dans ce cadre festif et rituel, on rejoint la valeur sociale et symbolique de la chasse et de la consommation de gibier qui s'est substituée à leur fonction strictement alimentaire. Peut-être ces festins aristocratiques accompagnés de beuveries rituelles étaient-ils, à Ugarit, l'occasion privilégiée où l'on consommait la venaison.

\section{LES ÉLÉMENTS DU DRAME}

Revenons au récit de la mort d'Aqhat, épisode central de ce qui nous reste de la Légende d'Aqhat. Le jeune héros est tué par un faucon envoyé par Anat en punition de son refus de lui donner son $\operatorname{arc}(K T U 1.17:$ vi $; 1.18$ : iv). Le récit ne donne aucune explication à l'insistance de la déesse pour obtenir du jeune homme son arc, alors qu'elle pourrait facilement s'en procurer un identique, comme

30. Les travaux relatifs aux confréries de marzihu sont nombreux; on se reportera aux plus récents : J. L. McLaughlin, «The marzeah at Ugarit. A Textual and Contextual Study », UF 23, 1991, p. 265-281; Dennis Pardee, «Marzihu, Kispu and the Ugaritic Funerary Cult : A Minimalist View », Ugarit, Religion and Culture. Essays Presented in Honour of Professor John C.L. Gibson, N. Wyatt - W. G. E. Watson - J. B. Lloyd (eds.), UBL 12, Münster, Ugarit-Verlag 1996, p. 273-287 ; Jean-Marie Husser, « À propos du festin marzihu à Ougarit: abus et impasse du comparatisme historique », dans F. Boespflug et F. Dunand (éd.), Le comparatisme en histoire des religions, Colloque international du Centre de Recherche d'Histoire des religions, Strasbourg 18-20 septembre 1996, Paris, Le Cerf, 1997, p. 158-173.

31. Je comprends la première ligne : il dbh. bbth. ms d. ș . bqrb/hklh « El offre dans sa maison un festin de chasse, de la venaison au milieu de son palais ». 
Aqhat le lui dit avec une certaine désinvolture. Ce point apparaît pourtant comme la clé du récit, car il implique la nature de la relation que la déesse cherchait à établir avec le héros et, par conséquent, la nature de la faute qui lui valut ce destin fatal. C'est donc l'ensemble des éléments du récit qu'il faut prendre en considération: 1) les circonstances du don de l'arc qui suscite la convoitise d'Anat; 2) les domaines de compétence de la déesse et leur signification dans le drame ; 3) la valeur symbolique de l'arc lui-même ; 4) les circonstances de la mort d'Aqhat enfin.

1) Dans le récit, le don d'un arc par Danil à son fils Aqhat marque l'accession de ce dernier à l'âge adulte (KTU 1.17 : v 33-39). La remise de ce cadeau est empreinte d'une certaine solennité et s'accompagne de recommandations paternelles. Avant de le donner à son fils, Danil bande lui-même l'arc ${ }^{32}$, geste qui veut signifier la transmission du savoir-faire qui accompagne l'objet, mais signale peut-être aussi que le garçon n'a pas encore toute la force nécessaire pour réaliser cette opération. La possession de cette arme et la pratique de la chasse qui lui est associée sont les signes et les moyens de son intégration dans l'aristocratie des hommes adultes. On a maintes fois souligné la valeur symbolique de cet arc, objet de prestige et signe d'appartenance à une catégorie sociale ${ }^{33}$. La description qui en est faite (KTU 1.17 : vi 20-25) l'identifie clairement comme un arc composite, arme qui s'introduit au Proche-Orient ancien et qui, associé à l'usage du char, révolutionne la pratique de la guerre dans le courant du Bronze moyen. Il s'agit non seulement d'une arme puissante, c'est aussi une arme précieuse en raison des matériaux utilisés, de la complexité de sa fabrication et de sa relative fragilité.

32. En traduisant un texte abîmé (KTU 1.17: v 34-36) avec Baruch Margalit (The Ugaritic Poem of AQHAT. Text, Translation, Commentary, BZAW 182, Berlin-New York, W. de Gruyter, 1989, p. 123 ; 177) : aphn . ǵzr . $m t$ / hrnmy . qšt . yqb .[ yš $] / r k \cdot{ }^{c} l$. aqht . $k y q\left[m{ }^{c} n\right]$ : «Alors le héros, l'homme de Harnam, l'arc, il le tend, [il le n]oue, près d'Aqhat il se tie[nt (pour) dire]... », suivent les paroles de recommandation. Autre proposition chez N. Wyatt, op. cit. (note 6), p. 269: «the bow he bent [and b]lessed ([yb]rk), on behalf of Aqhat indeed he ben[t it] (kyq[bh])».

33. Voir notamment P. Xella, op. cit. (note 2), p. 68 ; B. Margalit, op. cit. (note 32), p. 290. 
Cette grande valeur technologique et symbolique est signalée dans le récit par le fait que l'arc a été fabriqué par un dieu, l'artisan divin Kothar, également connu dans le mythe pour avoir produit les armes magiques qui ont permis à Baal de vaincre Yam.

En remettant l'arc à son fils, Danil lui fait la recommandation d'apporter «le meilleur/les prémices de ta chasse dans son/mon palais/temple ». Le texte est abîmé ${ }^{34}$ et ne permet pas de dire avec certitude qui est concerné par cette offrande : s'agit-il de l'offrande des prémices de la chasse dans le temple d'une divinité (cf. Gen 4,4), ou de l'approvisionnement du palais paternel avec le meilleurs de sa chasse (cf. Gen. 27,1-4) ? Le contexte incite généralement les traducteurs à préférer la première hypothèse, mais elle ne s'impose pas $^{35}$ et laisse de toute façon ouvert le choix de la divinité concernée par cette offrande de prémices : soit Kothar, en tant qu'auteur de cet arc (Wyatt), soit Anat, en sa qualité de chasseresse et de maîtresse des animaux (Caquot-Snycer-Herdner). Cette dernière interprétation rejoint sans doute la signification première de ce motif mythologique lorsqu'il apparaît dans un véritable mythe de chasse : le chasseur se doit d'honorer la divinité protectrice du gibier avec l'offrande d'une part de son butin. Mais comme on l'a dit plus haut, cet épisode fait désormais partie d'un ensemble narratif d'un autre genre et relève d'un contexte social et culturel différent. Quoi qu'il en soit, la scène évoque clairement un moment initiatique au sens large : le père remet à son fils devenu adulte un objet symbolique et lui enseigne un code de bonne conduite associé à la pratique de la chasse.

2) À côté de son caractère guerrier et de son activité de chasseresse, Anat est réputée pour sa beauté $\left(n^{c} m\right)$ et sa grâce $(t s m)^{36}$ et il

34. KTU 1.17: v 37-39:prcm $s s^{c} d k \cdot y b n[$ ] / prcm $\cdot s d k \cdot h n$ [ ] / șd . bhkly[ ]. Selon $K T U$, la lecture du dernier signe visible pourrait être $-y$, un suffixe $1^{\text {re }}$ personne qui réfère alors au locuteur, c-à-d Danil : «dans mon palais ». Selon CTA, ce signe pourrait être $-h$, un suffixe $3^{\mathrm{e}}$ personne, d'où la fréquente traduction « dans son palais $>$ dans son temple ».

35. On peut notamment hésiter sur la traduction de $h k l$ par « temple » ici. Le sens premier du terme est « palais », et il ne désigne la demeure d'une divinité que dans les mythes où elle est assimilée à un palais royal. En contexte cultuel, le temple est toujours désigné par $b t$. Le choix est également guidé par la lecture du suffixe, cf. note précédente.

36. $K T U 1.10$ : ii $16 ; 1.14$ : iii $41-42 ; 1.92: 27-30$. 
est assez généralement admis que la sexualité et l'érotisme font partie de ses attributions, à l'instar de l'Ishtar babylonienne. Pourtant, une définition fonctionnelle d'Anat comme déesse de l'amour apparaît trop simpliste et a été récemment contestée par les travaux de Peggy L. Day et de Neal H. Walls ${ }^{37}$, qui font justement remarquer qu'elle repose sur des bases textuelles fragiles. En tout état de cause, il est hors de doute qu'Anat se caractérise par une féminité particulière.

L'épithète batulatu, qui lui est presque exclusivement réservé dans les textes ougaritiques, la désigne nettement comme une très jeune femme, à peine nubile et pas encore mariée. Il ressort des recherches autour de ce terme que sa fréquente traduction par «vierge » ne rend pas exactement compte d'une signification qui réfère davantage au statut social de la jeune femme qu'à sa virginité physiologique ${ }^{38}$. Pour souligner l'intérêt de cet épithète dans notre propos, disons que batulatu renvoie nettement à une classe d'âge et implique une sexualité féminine encore adolescente, ainsi qu'un statut social encore mal défini quant au genre, notamment en raison du fait que la jeune femme ainsi désignée n'est pas encore mariée ou pas encore mère ${ }^{39}$. Ainsi, proche par son âge et son statut social des jeunes gens non mariés, Anat partage leur passion de la chasse et stimule leur courage dans le combat guerrier, dont elle incarne la fureur et la cruauté. On ne parlera donc pas de bisexualité à propos d'Anat, mais il est évident qu'elle assume la même ambiguïté sexuelle observée ailleurs chez Inanna-Ishtar ${ }^{40}$ ou l'Aphrodite chypriote ${ }^{41}$ : la sexualité d'une déesse féminine par excellence, mais au comportement parfois viril et occasionnellement affublée de traits masculins.

37. Peggy Day, «Why is Anat a warrior and hunter ?», dans W. Jobling et al. (eds), The Bible and the Politics of Exegesis, Cleveland, p. 141-156; «Anat : Ugarit's "Mistress of Animals" », JNES 51, 1992, p. 181-190 ; Neal H. Walls, The Goddess Anat in Ugaritic myth, SBLD 135, Atlanta, 1992.

38. Voir Clemens Locher, Die Ehre einer Frau in Israel, OBO 70, FreiburgGöttingen, Universität Verlag-Vandenhoeck \& Ruprecht, 1986.

39. Sur l'âge et la sexualité d'Anat, voir N. H. Walls, op. cit. (note 37), p. $78-86$.

40. Brigitte Groneberg, «Die sumerisch-akkadische Inanna-Ištar : Hermaphroditos ? »WO 17, 1986, p. 25-46.

41. Vinciane Pirenne-Delforge, L'Aphrodite grecque, Kernos Suppl. 4, Athènes-Liège, 1994, p. 348-353. 
3) L'insistance de la déesse à obtenir du jeune homme l'arc qu'il vient de recevoir en souligne la valeur symbolique. Dans le contexte où l'événement intervient, l'arc constitue à l'évidence un symbole de masculinité, et cette valeur symbolique excède la dimension de prestige social dont nous avons déjà parlé. Cela est si vrai que, pour mettre un terme à la discussion avec Anat et rendre son refus définitif, Aqhat lance à la déesse : «les arcs sont [pour] les guerriers, les femmes iraient-elles chasser maintenant? » (KTU 1.17 : vi 39-40). Visiblement, le jeune homme méconnaît les compétences de la déesse en ce domaine, compétences qu'elle ne manquera pas de lui rappeler un peu plus tard. Ce n'est donc pas le caractère sexiste de la réplique d'Aqhat qui provoque la colère de la déesse. La faute du héros ne réside pas non plus dans son scepticisme quant à la capacité de la déesse à lui procurer une forme de «vie éternelle» en échange, mais bien plutôt dans son refus d'accéder à une demande dont la nature exacte n'apparaît pas d'emblée.

Il est unanimement reconnu que cet épisode apparaît comme une reprise de celui où, dans l'Épopée de Gilgamesh, Ishtar offre au héros d'Uruk richesse et gloire en échange d'une relation amoureuse (Gilg. VI : i-ii) ${ }^{42}$, proposition qui se heurte au même refus méprisant du héros. L'analogie structurelle entre le refus de Gilgamesh de coucher avec Ishtar et le refus d'Aqhat de donner son arc à Anat autorise à considérer les deux récits comme des variantes d'un même motif mythologique ${ }^{43}$. La comparaison entre les deux mythes gagne encore en pertinence si l'on tient compte de la valeur symbolique

42. Texte de la version ninivite: Andrew R. George, The Babylonian Gilgamesh Epic, 2 vol., Oxford-New York, Oxford University Press, 2003 ; traduction française : Raymond J. Tournay - Aaron Shaffer, L'Épopée de Gilgamesh, LAPO 15, Paris, Le Cerf, 1994.

43. Pour le parallélisme entre Gilgamesh et Aqhat, voir Simon B. Parker, "Death and devotion: The composition and theme of Aqhat», dans Love and Death in the Ancient Near East. Essays in Honor of Marvin H. Pope, J.H. Marks, R. M. Good (eds), Guilford CT, Four Quarters, 1987, p. 71-83 ; N. H. Walls, op. cit. (note 37) p. 197-206 (bibliographie). La réaction d'Ishtar à l'humiliation que lui inflige le refus de Gilgamesh est également la même que celle d'Anat : l'une et l'autre montent au ciel vers le père des dieux pour réclamer l'autorisation de se venger du héros insolent (cf. Gilg. VI : ii 36 - iii 30 et $K T U 1.17:$ vi $46-18:$ i 19$)$. 
de l'arc d'Aqhat, substitué ici à la relation sexuelle demandée à Gilgamesh. Il est vrai que la question a été débattue de savoir si l'arc est bien ici un symbole sexuel ${ }^{44}$, ou s'il est à considérer strictement comme un symbole de masculinité, dépourvu de toute valeur sexuelle ${ }^{45}$. Cette dernière semble pourtant s'imposer du fait de la référence implicite à l'épisode parallèle de Gilgamesh, référence que les Ougaritains ne pouvaient pas ignorer. Cependant, la pleine signification de l'arc ne se réduit pas à cette dimension sexuelle et exige que l'on maintienne ensemble son double rapport à la masculinité et à la virilité. En sa qualité d'objet signifiant l'intégration du garçon au monde des hommes adultes, la possession de l'arc manifeste que Aqhat se conforme à la définition des genres telle qu'elle est admise dans la société dont il devient membre à part entière, et dans le même temps symbolise sa maturité sexuelle.

4) Dans une partie malheureusement très abîmée du texte (KTU $1.18:$ i) où seuls quelques mots subsistent à chaque ligne, on comprend que Anat attire Aqhat dans son piège en l'invitant à une partie de chasse, au prétexte, semble-t-il, de l'initier à cette pratique ${ }^{46}$. Le contexte initiatique de tout cet épisode se trouve ici confirmé, ainsi que le rôle d'Anat dans cette fonction. Mais s'agit-il uniquement d'une initiation à l'art cynégétique ? Dans le discours d'invitation que la déesse adresse au jeune homme intervient un vers passablement discuté : «Ecoute, je te [prie, va]leureux [Aqhat] : tu es mon frère et je [suis ta sœur]... ${ }^{47}$. L'interprétation classique de ce passage consiste à reconnaître dans les termes « frère » et « sœur » - ce dernier étant restitué - « commun expressions for lovers in the

44. Delbert R. Hillers, «The Bow of Aqhat : Meaning of a Mythological Theme », Orient and Occident, H. A. Hoffner (ed.), AOAT 22, KevelaerNeukirchen-Vluyn, Butzon \& Bercker-Neukirchener Verlag, 1973, p. 71-80 ; P. Xella, op. cit. (note 2), p. 69-71 ; N. H. Walls, op. cit. (note 37), p. 201 s.

45. Harold H. P. Dressler, "Is the bow of Aqhat a symbol of virility? » UF 7, 1975, p. 217-220 ; B. Margalit, op. cit. (note 32), p. 49-55 ; P. Day, op. cit. (note 37), p. 181-190.

46. KTU 1.18: i : ligne $27: l k t l k b s ̦ d[$ ]: «tu dois aller à la chasse [ ] »; ligne 29: almdk ș[d : «je t'apprendrai à ch[asser]».

47. $K T U 1.18:$ i $23-24: \check{S ̌ m}^{c} m\left[{ }^{c}\right.$ laqht ǵ]zr at ah̆ wan a[htk]. La restitution de la fin de la ligne 24 (a[htk]) est contestée par certains. 
Ancient Near East » ${ }^{48}$, ce qui ferait des paroles d'Anat une déclaration d'amour adressée au jeune homme, voir « a formal proposal of marriage on the part of Anatu ${ }^{49}$.

Après un examen rapide de la documentation proche-orientale, $H$. Dressler a remis en cause cette interprétation, constatant que ces valeurs pour «frère » et «sœur » ne sont attestées que dans la poésie amoureuse sumérienne et égyptienne. Refusant la restitution $a[h t k]$ « ta sœur », en fin de ligne, il considère ces paroles comme le début de l'invitation à la chasse : «Come, o brother, and myself will $[\ldots]^{50}$. D'autres maintiennent le parallélisme « mon frère » // «ta sœur», mais voient dans ces termes soit l'expression d'une égalité de rang entre la déesse et le jeune prince, en référence à l'usage épistolaire et au langage diplomatique ${ }^{51}$, soit une déclaration feinte de réconciliation dans le but de l'attirer dans une embuscade $^{52}$. Il est difficile d'opter pour une solution définitive dans un texte irrémédiablement abîmé. Remarquons aussi que les objections de Dressler ne sont pas si contraignantes qu'il le prétend, car l'usage de «sœur» pour désigner la fiancée se retrouve bien dans la Bible (Cant. 4,9-10); si l'on maintient la restitution vraisemblable de «ta sœur», la question reste donc ouverte. L'interprétation sexuelle de ces termes implique que l'initiation à la chasse s'accompagnait également d'une initiation à la sexualité, l'une et l'autre sous l'égide d'Anat. Sans être assurée d'un point de vue textuel, cette éventualité serait dans la logique de ce que nous venons de voir à propos de la symbolique sexuelle de l'arc, sur la propriété duquel la déesse exprime sa revendication.

48. Theodore H. Gaster, Thespis, New York, Schuman, 1950, p. 290.

49. Meindert Dijkstra, Johan C. de Moor, "Problematical Passages in the Legend of Aqhâtu", $U F$ 7, 1975, p. 194.

50. Harold H. P. Dressler, "The Metamorphosis of a Lacuna. Is at.ah.wan... a Proposal of Marriage ?" UF 11, 1979, p. 211-217» ; cette lecture est reprise par N. Wyatt, op. cit. (note 6), p. 279.

51. Paolo Xella, « Tu sei mio fratello ed io sono tua sorella (KTU 1.18 I 24) », AuOr 2, 1984, p. 151-153.

52. Dennis Pardee, «The Aqhatu Legend », dans W. W. Hallo (ed.), The Context of Scripture, vol. 1, Canonical Compositions from the Biblical World, Leiden-New York, Brill, 1997, p. 348. 
L'invitation d'Anat à venir chasser avec elle n'est finalement qu'une feinte pour attirer le jeune homme dans un piège, et il apparaît d'emblée que le rôle de ce dernier dans le jeu ne sera pas celui du chasseur, mais de la proie. Les circonstances de la mort d'Aqhat illustrent ainsi de façon exemplaire la réversibilité du jeu cynégétique dont il a été question en introduction. Pour exécuter son plan meurtrier, Anat a recours aux services d'un mercenaire, Yatipanu, personnage qu'elle métamorphose en faucon pour en faire une arme mortelle ${ }^{53}$. Elle-même plane tel un oiseau au-dessus du jeune homme en train de prendre son repas, harnachée comme un chasseur. On a depuis longtemps reconnu dans ce texte la référence à une technique de fauconnerie ${ }^{54}$ qui souligne encore la transformation d'Aqhat en animal traqué par son prédateur. Le rapprochement a été fait avec le mythe d'Actéon, dont le nom même pourrait être une hellénisation du cananéen Aqhat ${ }^{55}$ : la mort du héros grec, transformé en cerf par Artémis et dévoré par ses propres chiens, offre un parallèle précis à cette inversion des rôles du chasseur et de la proie.

\section{ANAT, DIVINITÉ PRÉSIDANT À L'INITIATION DES JEUNES GENS}

La prise en compte de la dimension symbolique de l'arc permet de décoder la nature de la demande d'Anat, mais l'ambivalence des symboles ouvre plusieurs possibilités non exclusives. Selon l'une d'elles, Anat exige du jeune homme un abandon symbolique de sa

53. KTU 1.18 : iv 27-29 : tqh yṭpn mhr št / tštn knšr bhbšh km diy / bt' rth : « elle prend Yatipan le mercenaire, elle le met comme un faucon à sa ceinture, comme un rapace sur son gant $»$.

54. Wilfred G. E. Watson, «The Falcon Episode in the Aqhat Tale », JNSL 5, 1977, p. 71-72. Cette interprétation, très généralement acceptée, a été critiquée par Karin Reiter, « Falknerei im Alten Orient ? Die Quellen », MDOG 121, 1989, 169-196 ; et «Falkenrei in Ugarit», UF 22, 1990, p. 271-278. La pratique de la fauconnerie est maintenant bien attestée dans le monde hittite ; voir Jeanny V. Canby, «Falconry (Hawking) in Hittite Lands », JNES 61, 2002, p. 161-201, qui commente un dossier iconographique très convaincant.

55. Selon une hypothèse de Michael C. Astour, Hellenosemitica: An Ethnic and Cultural Study in West Semitic Impact on Mycenaean Greece, Leiden, Brill, 1965., p. 163-168. 
virilité, abandon qui pourrait s'entendre comme un retour momentané et ritualisé du jeune homme à l'état asexué de l'enfance, voire comme un échange de sexe avec la déesse qui, on l'a dit, assume volontiers un comportement masculin ${ }^{56}$. Autre possibilité : la déesse propose au jeune homme, sur le mode de la métaphore, une relation sexuelle, comme le fait Ishtar à Gilgamesh. Cette offre signifierait, pour le jeune homme, une double initiation : à la sexualité d'une part et à la pratique de la chasse d'autre part, deux activités caractéristiques d'un homme adulte et qui relèvent toutes deux du champ des compétences d'Anat. Cet aspect se trouve éventuellement confirmé par les paroles d'Anat discutées ci-dessus. Abandon symbolique de sa virilité, échange ritualisé des genres avec la déesse ou initiation à la sexualité, cette demande faite au garçon parvenant à l'âge adulte par une déesse « vierge » au comportement viril apparaît certes comme une mise à l'épreuve, mais elle évoque surtout cette inversion des signes si caractéristiques des rites de passage et de puberté 57 .

Envisager l'implication d'Anat dans un rite de passage des jeunes gens à l'âge adulte ne contrevient nullement à ce que nous savons par ailleurs de cette divinité ${ }^{58}$. Comme on l'a dit plus haut, Anat est pleinement féminine quant à sa sexualité, mais ambiguë quant au genre, susceptible à ce titre de transgresser les codes sociaux qui le définissent. Neal $\mathrm{H}$. Walls note très justement que Aqhat « is a victim of Anat's confused gender and sexual identity ${ }^{59}$. De la sorte, au moment crucial où le garçon va assumer définitivement sa masculinité parmi les hommes adultes, l'intervention de la déesse

56. On ne parlera cependant pas ici de castration symbolique comme l'a fait D. R. Hillers (op. cit. note 44) dans une interprétation freudienne de ce passage.

57. Voir sur ce point Angelo Brelich, Paides e Parthenoi, Roma, 1969, p. 157-166 ; P. Vidal-Naquet, op. cit. (note 11), p. 163 s., qui note : «... le passage de l'enfance à l'âge adulte, celui de la guerre et du mariage, est dramatisé, dans le rite et dans le mythe, au moyen d'une loi qu'on pourrait appeler loi d'inversion symétrique ».

58. Où Anat apparaît comparable à Artémis dans sa fonction de courotrophe. Pour un rôle analogue d'Aphrodite, associée à Hermes dans un contexte initiatique au sanctuaire de Kato Syme en Crète, voir N. Marinatos, op. cit. (note 12).

59. N. H. Walls, op. cit. (note 37), p. 204. 
opère comme médiateur symbolique : elle propose au jeune héros une relation où l'érotisme et la sexualité restent camouflés sous l'apparence virile d'un partenariat de chasse. Comme chasseresse et guerrière, Anat inverse les rôles assignés à l'homme et à la femme dans la société et, par cette double transgression - à l'égard de la féminité comme à l'égard de la pratique cynégétique - elle incarne pour le jeune homme une sorte d'absolu de la virilité guerrière à laquelle il accède ${ }^{60}$. Il apparaît bien que le ressort de l'intrigue réside précisément dans l'incapacité d'Aqhat à déchiffrer cette situation et l'ambivalence de son rapport à la déesse. Son refus de lui donner son arc doit donc s'entendre comme une faute rituelle : il signifie sa méconnaissance d'un code symbolique propre à signifier et à structurer son passage à l'âge adulte et son agrégation au monde des hommes. De ce point de vue, l'épisode de la mort d'Aqhat est le récit paradigmatique de l'échec de ce passage pour quiconque refuse le code social définissant les rapports entre les sexes et l'autorité de la déesse en la matière.

On l'a dit, la valeur symbolique de l'arc d'Aqhat se comprend également dans un usage métaphorique du vocabulaire cynégétique pour signifier la quête sexuelle ${ }^{61}$. L'exercice cynégétique sert ici

60. Il faut rappeler ici les lignes fameuses de Jean-Pierre Vernant dans Mythe et société en Grèce ancienne, Paris, Maspéro, 1979, p. 37 s. : «Le mariage est à la fille ce que la guerre est au garçon : pour tous les deux, ils marquent l'accomplissement de leur nature respective, au sortir d'un état où chacun participe encore de l'autre. Aussi une fille qui se refuse au mariage, renonçant du même coup à sa "féminité", se trouve-t-elle en quelque sorte rejetée du côté de la guerre pour devenir paradoxalement équivalente à un guerrier. C'est ce que l'on constate, sur le plan du mythe, avec des personnages féminins du type des Amazones et, sur le plan religieux, avec des déesses comme Athéna : leur statut de guerrière est lié à leur condition de parthenos ayant fait vœu à jamais de virginité. On peut même dire que cette déviance par rapport à l'état normal de la femme, comme par rapport à l'état normal du guerrier, réservé à l'homme, non à la femme, donne aux valeurs guerrières, dès lors qu'elles sont incarnées par une fille, leur maximum d'intensité. D'une certaine façon elles cessent d'être relatives et limitées à un sexe pour devenir "totales". » Je pense que ces considérations sont pour une large part extensibles à la culture syrienne du Bronze récent.

61. Voir Jean-Marie Husser, « Chasse et érotisme dans les mythes ougaritiques », Ktema 33, 2008, p. 235-244. 
tout à la fois d'épreuve initiatique et de métaphore au jeu érotique. Dans ce langage, la femme, tout comme le gibier pour le chasseur, est l'objet d'un désir intense qui, dans les deux situations, se dit par le même verbe $h m d$, «désirer ardemment ». Un petit récit mythologique racontant une chasse d'Ashtart en offre la meilleure illustration $(K T U 1.92)^{62}$. Dans ce récit, la déesse assume successivement deux positions : elle apparaît tout d'abord comme chasseresse à l'affût d'un taureau et convoitant sa proie dans les contrées sauvages. Dans ce qui semble être la seconde partie du récit, elle devient à son tour, dans un vignoble, objet de la convoitise sexuelle de Baal. L'usage du même verbe ḥmd, et le fait que Baal apparaît affublé de cornes, suggèrent un véritable effet de miroir. Dans ce cadre général d'une opposition entre l'espace sauvage de la chasse et l'espace clos des jardins, le récit est construit sur la symétrie des situations et la réversibilité du jeu cynégétique : successivement sujet et objet du désir, Ashtart apparaît comme prédatrice et comme proie, tandis que le taureau qu'elle abat dans la cannaie est désigné par l'épithète $b a^{c} a l u^{63}$, et que c'est un Baal porteur de cornes qui la poursuit de ses assiduités.

Avec la métaphore cynégétique pour dire un aspect des rapports entre les sexes, et la réversibilité des situations entre le chasseur et sa proie, nous tenons la clé de la relation entre Aqhat et Anat dans ce drame. Quand, par mésaventure, c'est le héros qui est tué, chasseur devenu proie à son tour, ne se trouve-t-il pas dans la situation de la femme « chassée » par l'homme ? C'est selon cette équation métaphorique qu'il faut lire la mort d'Aqhat, lui qui fut tué lors d'une partie de chasse par un faucon envoyé par Anat chasseresse. Sa mort inverse les rôles dans la partie de chasse : de chasseur il est réduit à la position de gibier; mais elle signifie également une inversion symbolique des sexes entre lui et la déesse : il est devenu la femme (la proie), tandis que la déesse assume le rôle du chasseur, autrement dit, de l'homme qu'Aqhat n'a pas su devenir. Si sa mort

62. Traductions : A. Caquot, op. cit. (note 29), p. 31-36 ; N. Wyatt, op. cit. (note 6), p. 370-374.

63. KTU 1.92 : 13-14 : tr̂mỹ ltr tšpl bcl cbb : «elle ti[re] sur le taureau, elle abat le seigneur assoiffé ». 
sanctionne une faute survenue lors de son passage à l'âge adulte, il faut y lire l'expression symbolique de son échec à intégrer le monde des hommes : sa régression au rôle de proie signifie qu'il est maintenu dans la sphère du féminin, c'est-à-dire dans le monde de l'enfance, au moment même où il devait accéder au monde des hommes adultes.

L'état fragmentaire du texte ne permet pas de préciser le détail des éventuels rites accompagnant ce passage à l'âge adulte, ni la nature exacte de l'échec d'Aqhat. Quoi qu'il en soit, cette lecture d'un épisode bien connu de la Légende d'Aqhat me semble fournir suffisamment d'indices pour conclure à l'existence, dans la classe dominante à Ugarit, d'un code symbolique et, selon toute vraisemblance, d'un rituel associés à la pratique de la chasse. Ce code et ce rituel visaient à structurer, sous le patronage de la déesse Anat, le passage des garçons dans le monde des hommes adultes, à travers une initiation à la pratique de la chasse et peut-être aussi de la sexualité.

Université de Strasbourg

UFR des Sciences historiques - UMR 7044

9, place de l'Université

67084 Strasbourg

husser@umb.u-strasbg.fr 